\title{
Silver nanoparticle production by the fungus Fusarium oxysporum: nanoparticle characterisation and analysis of antifungal activity against pathogenic yeasts
}

\author{
Kelly Ishida ${ }^{1}{ }^{+}$, Talita Ferreira Cipriano ${ }^{2}$, Gustavo Miranda Rocha ${ }^{3,4}$, \\ Gilberto Weissmüller ${ }^{3}$, Fabio Gomes ${ }^{5}$, Kildare Miranda ${ }^{5}$, Sonia Rozental ${ }^{2}$
}

\begin{abstract}
'Laboratório de Quimioterapia Antifúngica, Departamento de Microbiologia, Instituto de Ciências Biomédicas, Universidade de São Paulo, São Paulo, SP, Brasil ${ }^{2}$ Laboratório de Biologia Celular de Fungos ${ }^{3}$ Laboratório de Física Biológica ${ }^{5}$ Laboratório de Ultraestrutura Celular Hertha Meyer, Instituto de Biofísica Carlos Chagas Filho, Universidade Federal do Rio de Janeiro, Rio de Janeiro, RJ, Brasil ${ }^{4}$ Instituto Nacional de Metrologia, Padronização e Qualidade Industrial, Duque de Caxias, RJ, Brasil
\end{abstract}

The microbial synthesis of nanoparticles is a green chemistry approach that combines nanotechnology and microbial biotechnology. The aim of this study was to obtain silver nanoparticles (SNPs) using aqueous extract from the filamentous fungus Fusarium oxysporum as an alternative to chemical procedures and to evaluate its antifungal activity. SNPs production increased in a concentration-dependent way up to $1 \mathrm{mM}$ silver nitrate until 30 days of reaction. Monodispersed and spherical SNPs were predominantly produced. After 60 days, it was possible to observe degenerated SNPS with in additional needle morphology. The SNPS showed a high antifungal activity against Candida and Cryptococcus, with minimum inhibitory concentration values $\leq 1.68 \mu \mathrm{g} / \mathrm{mL}$ for both genera. Morphological alterations of Cryptococcus neoformans treated with SNPs were observed such as disruption of the cell wall and cytoplasmic membrane and lost of the cytoplasm content. This work revealed that SNPs can be easily produced by $\mathrm{F}$. oxysporum aqueous extracts and may be a feasible, low-cost, environmentally friendly method for generating stable and uniformly sized SNPs. Finally, we have demonstrated that these SNPs are active against pathogenic fungi, such as Candida and Cryptococcus.

Key words: silver nanoparticle - microscopy - environmentally friendly procedure - antifungal - Candida - Cryptococcus

Nanotechnology is a rapidly expanding field and have been potentially used in a wide assortment of commercial products worldwide. Silver nanoparticles (SNPs) have attracted specific attention due to their potential use in a range of applications, such as electronics, biosensing, clothing manufacture, food storage, paints, sunscreens, cosmetics and medical devices (Ahamed et al. 2010). SNPs have also a potent antibacterial and antifungal activity and general anti-inflammatory effects. Furthermore, SNPs can improve wound healing and may be exploited to develop dressings for wounds and burns and antibacterial coatings on medical devices to reduce nosocomial infection rates (Ahamed et al. 2010, Chaloupka et al. 2010).

Although ultraviolet irradiation, aerosol technologies, lithography, laser ablation, ultrasonic fields and photochemical reduction techniques have been used successfully to produce nanoparticles, they remain expensive and involve the use of hazardous chemicals (Narayanan \& Sakthivel 2010). Therefore, there is a growing effort

doi: $10.1590 / 0074-0276130269$

Financial support: CNPq, CAPES, FAPERJ

+ Corresponding author: ishidakelly@usp.br

Received 17 May 2013

Accepted 16 September 2013 to develop environmentally friendly methods for nanoparticle production. The microbial synthesis of nanoparticles is a prime candidate approach because it is a green chemistry method that combines nanotechnology and microbial biotechnology (Narayanan \& Sakthivel 2010). Some microorganisms, including bacteria, yeast and filamentous fungi play an important role in the remediation of toxic metals through the reduction of the metal ions; therefore, these microorganisms could be employed as nanofactories for nanoparticle production (Fortin \& Beveridge 2000). Several studies have shown that metal nanoparticles, such as gold, silver, gold-silver alloy, selenium, tellurium, platinum, palladium, silica, titanium, zirconium, quantum dots and magnetite can be biosynthesised by bacteria, actinomycetes, fungi and viruses [revised by Narayanan and Sakthivel (2010)].

The fungus Fusarium oxysporum can reduce aqueous silver ions extracellularly to generate SNPs (Ahmad et al. 2003). This process likely occurs through the action of both reductase enzymes and electron shuttle quinones (Durán et al. 2005). In addition, biological nanoparticle synthesis often yields a more consistent size distribution pattern than other methods due to direct stabilisation of the nanoparticles by proteins involved in the synthesis process, as described by Durán et al. (2005).

Fusarium spp are filamentous fungi that are widely distributed in soil, water, subterranean and aerial plant parts, plant debris and other organic substrates (Nelson et al. 1994). The widespread distribution of Fusarium spp is attributed to their ability to grow on a wide range 
of substrates and their efficient dispersal mechanisms (Burgess 1981). Most species are harmless saprobes and members of the soil microbial community; however, many species are mycotoxin producers and are pathogenic to plants and humans.

In the present paper, we investigate the kinetics of SNPs production using an environmentally friendly method of extracellular biosynthesis by $F$. oxysporum. The characteristics and stability of the SNPs were evaluated by electron and atomic force microscopy and the antifungal activity against pathogenic species of Candida and Cryptococcus was evaluated by broth microdilution and agar diffusion tests.

\section{MATERIALS AND METHODS}

Fungal strains - The F. oxysporum 07 SD strain was kindly provided by Elisa Espósito (Center for Environmental Sciences, University of Mogi das Cruzes, Mogi das Cruzes, state of São Paulo, Brazil). The fungus was cultivated and maintained on potato dextrose agar (PDA) (Becton \& Dickinson and Company, USA) at $28^{\circ} \mathrm{C}$ in Petri dishes.

The following strains of Candida spp and Cryptococ$c u$ spp were used to evaluate the antifungal activity of the SNPs: Candida albicans ATCC 10231, C. albicans ATCC 24433, Candida krusei ATCC 6258, Candida glabrata ATCC 2001, Candida parapsilosis ATCC 22019, Candida tropicalis ATCC 13803, Cryptococcus neoformans ATCC 28957 and Cryptococcus gattii ATCC 56990. The yeasts were maintained on Sabouraud dextrose agar (SDA) (Becton \& Dickinson) at $4^{\circ} \mathrm{C}$ and subcultured at least twice on the same medium at $35^{\circ} \mathrm{C}$ for $48 \mathrm{~h}$ prior to use in experiments to ensure optimal growth.

SNP production - F. oxysporum previously grown on PDA at $28^{\circ} \mathrm{C}$ was inoculated in medium containing $2 \%$ malt extract and $0.5 \%$ yeast extract and incubated at $28^{\circ} \mathrm{C}$ for 7 days with agitation. Subsequently, the biomass was centrifuged, washed three times with sterile water and weighed. Approximately $10 \mathrm{~g}$ of $F$. oxysporum biomass was added to a glass Erlenmeyer flask containing $100 \mathrm{~mL}$ distilled water and incubated for $72 \mathrm{~h}$ at $28^{\circ} \mathrm{C}$ with agitation. Then, the components of the fungal aqueous extract were obtained by filtration through a $0.45 \mu \mathrm{m}$ pore size nylon membrane filter and a silver nitrate $\left(\mathrm{AgNO}_{3}\right)$ solution was added to produce different concentrations of silver ions $(0.5 \mathrm{mM}, 1.0 \mathrm{mM}, 1.5 \mathrm{mM}$ and $2.0 \mathrm{mM}$ ). The solutions were kept up to 60 days at room temperature in the dark. Periodically, aliquots of the reaction solutions were removed and the absorption was measured from 200-600 $\mathrm{nm}$ with a spectrophotometer (SpectraMax M2e, Molecular Devices, Sunnyvale, CA, USA). The aliquots were also characterised using microscopy methods as follows.

SNP characterisation - Transmission electron microscopy (TEM) - A drop of SNP colloidal solution obtained from a $1.0 \mathrm{mM} \mathrm{AgNO}_{3}$ solution collected at various times $(5,10,15,30$ and 60 days) was directly dispensed on a copper grid (300 mesh) coated with polyvinyl resin (FormVar, Ted Pella, Inc) and dried in a vacuum desiccator. Images of the SNPs were obtained with a Jeol 1200 electron mi- croscope (Jeol, Tokyo, Japan) equipped with a CCD Camera (MegaView III model, Soft Image System, Germany) and processed with iTEM software (Soft Image System). Routines were performed following the guidelines established by National Institute of Standards and Technology (NIST) for size characterisation by microscopy-based techniques (Jillavenkatesa et al. 2001). The SNPs size was measured using the software ImageJ [National Institutes of Health (NIH), USA] (rsb.info.nih.gov/ij/) from several images obtained by TEM. For elemental analysis, energy dispersive X-ray spectroscopy (EDX) was performed. Briefly, X-rays were collected using a lithium-drifet silicon detector with a Norvar window in a $0-10 \mathrm{keV}$ energy range with a resolution of $10 \mathrm{eV} /$ channel.

Scanning electron microscopy (SEM) - A drop of SNP colloidal solution obtained from a $1.0 \mathrm{mM} \mathrm{AgNO}$ solution at various days of incubation $(5,10,15,30$ and 60 days) was directly adhered to coverslips coated with FormVar and subsequently recovered by a carbon strip. The nanoparticles were analysed by a backscattered electron detector with a QUANTA 250 scanning electron microscope (FEI Company, Tokyo, Japan) operating at high vacuum mode.

Atomic force microscopy - A $10 \mu \mathrm{L}$ aliquot of SNP colloidal solution obtained from a $1.0 \mathrm{mM} \mathrm{AgNO}$ solution at various times $(5,10,15,30$ and 60 days) was spread onto freshly cleaved $1 \mathrm{~cm}^{2}$ mica slides and dried with a low pressure jet of nitrogen gas. Next, the SNPs on the slide were examined by an MFP-3D-BIO system (Asylum Research, Santa Barbara, CA, USA) operating in intermittent contact mode using a cantilever Olympus AC240TS. SNP size analyses were quantified and diameter size measured with the package particle analysis function in ImageJ (NIH) using several SNPs images according to standards established by NIST (Jillavenkatesa et al. 2001).

Antifungal activity assay - The in vitro antifungal activity of the SNPs was evaluated using the disk difusion method (CLSI 2004) with some modifications. This assay was used to test SNPs formed with different concentrations of $\mathrm{AgNO}_{3}$ solutions and at different time points after the start of the reaction. Briefly, Müller-Hinton agar (Becton, Dickinson and Company) Petri plates (4 $\mathrm{mm}$ of depth) were prepared and, subsequently, $100 \mu \mathrm{L}$ of fungal inoculum [1-5 x $10^{6}$ colony-forming unit $\left.(\mathrm{CFU}) / \mathrm{mL}\right]$ was uniformly spread onto the plates. Then, a $5 \mu \mathrm{L}$ aliquot of SNP colloidal solution was impregnated on Whatman No. 1 sterile filter paper discs ( $5 \mathrm{~mm}$ in diameter). The discs were applied to the plates, which were then incubated at $35^{\circ} \mathrm{C}$ for $24-48 \mathrm{~h}$. Finally, the inhibition halo was measured. Water and aqueous fungal extract were used as negative controls for antifungal activity.

In addition, the antifungal activity of the SNP colloidal solution obtained from a 30-day reaction with $1.0 \mathrm{mM} \mathrm{AgNO}_{3}$ solution was evaluated by the broth microdilution method (CLSI 2008). Briefly, the SNPs were serially diluted two-fold in RPMI-1640 medium without sodium bicarbonate (Sigma Chemical Co, MO, USA) buffered with 0.165 M MOPS (Sigma Chemical 
Co) in 96-well microtitre trays to obtain concentrations of 0.1 to $54 \mu \mathrm{g} / \mathrm{mL}$. A fungal suspension of $1-5 \times 10^{3}$ $\mathrm{CFU} / \mathrm{mL}$ was prepared and $100 \mu \mathrm{L}$ was dispensed into each well to obtain final concentrations of $0.5-2.5 \times 10^{3}$ $\mathrm{CFU} / \mathrm{mL}$. The microtitre trays were incubated at $35^{\circ} \mathrm{C}$ for $48 \mathrm{~h}$ (Candida spp) or $72 \mathrm{~h}$ (Cryptococcus spp) in a humidified chamber. The minimum inhibitory concentration (MIC) was the lowest concentration of SNPs that resulted in visual inhibition of fungal growth.

The determination of the minimum fungicidal concentration (MFC) was performed after $48 \mathrm{~h}$ of treatment with the inhibitory concentrations used in the broth microdilution assay. An aliquot of all treatments were transferred onto SDA plates without the presence of drugs. The plates were incubated at $35^{\circ} \mathrm{C}$ for $48 \mathrm{~h}$ and the MFC was determined. MFC means the lower concentration which showed no fungal growth.

Morphological alterations - C. neoformans ATCC 28957 yeasts were selected for the following experiments due to higher antifungal susceptibility presenting the lowest SNPs MIC and MFC values. Yeasts were treated with a sub-inhibitory concentration $(0.21 \mu \mathrm{g} / \mathrm{mL})$ of SNPs, for $72 \mathrm{~h}$ at $35^{\circ} \mathrm{C}$, were washed in PBS, $\mathrm{pH} 7.2$ and fixed in $2.5 \%$ glutaraldehyde and $4 \%$ paraformaldehyde in $0.1 \mathrm{M}$ cacodylate buffer. Then, the yeasts were post-fixed in $1 \%$ osmium tetroxide in cacodylate buffer containing $1.25 \%$ potassium ferrocyanide and $5 \mathrm{mM}$ $\mathrm{CaCl}_{2}$ for $2 \mathrm{~h}$ and serially dehydrated in ethanol. Subsequently, the yeasts were embedded in Spurr's epoxy resin and ultra-thin sections were obtained using a ReichertJung Ultracut (Leica Microsystems, Wetzlar, Germany). Finally, the sections were stained with $5 \%$ uranyl acetate and $0.5 \%$ lead citrate and visualised on a Jeol 1200 electron microscope.

Statistical analysis - Statistical analyses were performed using Prism 5.0. One-way ANOVA (Dunnett's test) was utilised to compare the SNP production from different silver concentrations and Student's $t$ test was used to analyse the SNP size. Samples were considered statistically significant when $\mathrm{p}<0.05$.

\section{RESULTS}

SNP production - The production of SNPs was initially evaluated visually for up to 60 days during the reaction of silver ions and aqueous extracts from $F$. oxysporum. Darkening of the medium colour occurred during the 60 days of the reaction for different silver ion concentrations compared to aqueous extract from $F$. oxysporum without $\mathrm{AgNO}_{3}$ solution and sterile distilled water. The darkening of the colloidal solution occurred in a time-dependent manner for the first five days of the reaction; after five days, the colour remained constant until 60 days (data not shown). These colour changes are related to the reduction of silver ions that occurs during the production of SNPs. At all this reaction times, the colour of the colloidal solutions became darker in a dose-dependent manner until the concentration of $1.5 \mathrm{mM} \mathrm{AgNO}$. SNP production was quantitatively measured by spectrophotometry. SNPs exhibited the highest absorbance between 340-560 $\mathrm{nm}$, with an absorption peak at $440 \mathrm{~nm}$, for all $\mathrm{AgNO}_{3}$ concentrations used and reaction times tested (Fig. 1). Fig. 2 shows that SNP production proceed the fastest rate from zero-three days; production continued to increase up to 30 days and decrease between 30-60 days. Furthermore, SNP production increased dose-dependently way to the silver ion concentration added to the reaction (Fig. 2). Significantly fewer SNPs were produced from the 0.5 $\mathrm{mM}$ solution, as evaluated by absorbance at $440 \mathrm{~nm}$, than from ion silver concentrations above $1 \mathrm{mM}(\mathrm{p}<0.01$, one-way ANOVA); this difference was most striking on the third day of the reactions ( $p<0.001$, one-way ANOVA). Interestingly, there was no statistically significant difference in SNP production at concentrations above 1 $\mathrm{mM} \mathrm{AgNO}$. For this reason, we used $1 \mathrm{mM} \mathrm{AgNO}_{3}$ solutions for SNP production in the following experiments characterising SNPs.

SNPs characterisation - Several microscopy techniques were used to confirm SNP production, identify the presence of silver atoms and analyze SNP size and morphology. TEM micrographs of five days SNPs revealed the presence of spherical electron-dense nanoparticles that were frequently monodispersed and relatively homogeneous in size, with variations in diameter from 3.4-26.8 $\mathrm{nm}$ (Fig. 3A, Table I). Maximum SNP diameter $(64.9 \mathrm{~nm})$ was observed on the 30th day (Fig. 3B, Table I).

After 60 days of reaction, we observed needle-shaped nanoparticles in addition to the spherical form previously observed. In addition, the spherical SNP diameter was very heterogeneous varying from 1.9-53.6 nm (Fig. 3C, Table I). There was observed a significant difference in SNP size between all reactions times examined $(\mathrm{p}<$ 0.01 ), except between five-15 days. However, it is important to note that at five days, the SNPs were more homogeneous in size than in 15 days and generally more homogenous dispersed from each other. The presence of silver atoms in the colloidal solution was confirmed by EDX (black arrow in Fig. 3D).

Morphological analysis by AFM revealed two distinct structural arrangements of SNPs: one of $300 \pm 57 \mathrm{~nm}$ and another of $77 \pm 30 \mathrm{~nm}$ (Fig. 4B). The smaller structures observed by AFM include the spherical electron-dense SNPs observed by TEM. The AFM images demonstrate that the particle sizes are homogeneously distributed over the mica. In addition, medium-sized $(50 \mathrm{~nm})$ SNPs were detected by SEM with a backscattered electron detector (Fig. 4A). We also observed an amorphous material surrounding some groups of nanoparticles.

Antifungal activity - All Candida species tested were susceptible to the SNPs produced from $\mathrm{AgNO}_{3}$ solutions with concentrations above $1 \mathrm{mM}$. The results from the agar diffusion method revealed that the inhibition halo diameter was similar for SNPs collected between five60 days of reaction (Table II). Among Candida species, C. albicans ATCC 10231 was the most susceptible to SNPs, as indicated by a growth inhibition halo of $13 \mathrm{~mm}$ when incubated with SNPs produced from $1 \mathrm{mM} \mathrm{AgNO}_{3}$ $(\sim 0.54 \mu \mathrm{g})$. By contrast, C. krusei ATCC 2001 and $C^{3}$. tropicalis ATCC 13803 were less susceptible to SNPs. Similar patterns of growth inhibition were observed for the Cryptococcus species (Table II). 
The broth microdilution method revealed that the Cryptococcus species were slightly more susceptible to SNP treatment (MICs from 0.42-0.84 $\mu \mathrm{g} / \mathrm{mL}$ ) than the Candida species (MICs from 0.84-1.68 $\mu \mathrm{g} / \mathrm{mL}$ ) (Table III). In addition, SNPs exhibited fungicidal activity (with MFC values up to 4 times the MIC value) against most of the species tested, with the exception of $C$. parapsilosis ATCC 22019 and C. krusei ATCC 6258 (Table III). These results are in agreement with those obtained using the agar diffusion method.

Morphological alterations of C. neoformans treated with SNPS - Untreated C. neoformans ATCC 28957 yeasts exhibited a well-preserved cellular ultrastructure with a compact cell wall (CW), continuous cytoplasmic membrane, homogeneous and electron-dense cytoplasm and polysaccharide capsule (Fig. 5A, B). By contrast, yeasts treated with sub-inhibitory concentrations of SNPs $(0.21$ $\mu \mathrm{g} / \mathrm{mL}$ ), for $72 \mathrm{~h}$ at $35^{\circ} \mathrm{C}$, exhibited a disrupted $\mathrm{CW}$ and several invaginations in the cytoplasmic membrane (Fig. 5C, D). In addition, increased $\mathrm{CW}$ thickness was observed (Fig. 5E, F). Interestingly, some SNPs appear to be retained in the fungal capsule (black arrowhead in Fig. 5F).

\section{DISCUSSION}

In the last decade, the development of biological systems as an environmentally friendly method for metal nanoparticle formation has emerged as an interesting and important scientific field. A wide number of micro- organisms, including bacteria, yeast, filamentous fungi, algae and plants, have been shown to be capable of fabricating various types of metal nanoparticles like silver, gold, palladium and others (Quester et al. 2013).

Previous studies have demonstrated that filamentous fungi, such as F. oxysporum (Durán et al. 2005), Fusarium accuminatum (Ingle et al. 2008), Aspergillus niger (Gade et al. 2008), Amylomyces rouxii (Musarrat et al. 2010) and the endophytic fungus Epicoccum nigrum

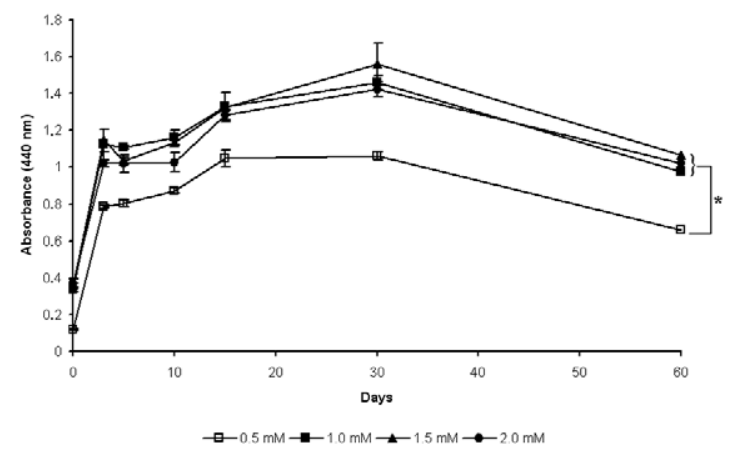

Fig. 2: extracellular silver nanoparticles (SNPs) production by silver nitrate solutions mixed with aqueous extract from Fusarium oxysporum over time (up to 60 days). The absorption of the SNPs was measured at $440 \mathrm{~nm} . *$ : $\mathrm{p}<0.05 ;{ }^{* *}: \mathrm{p}<0.01$.
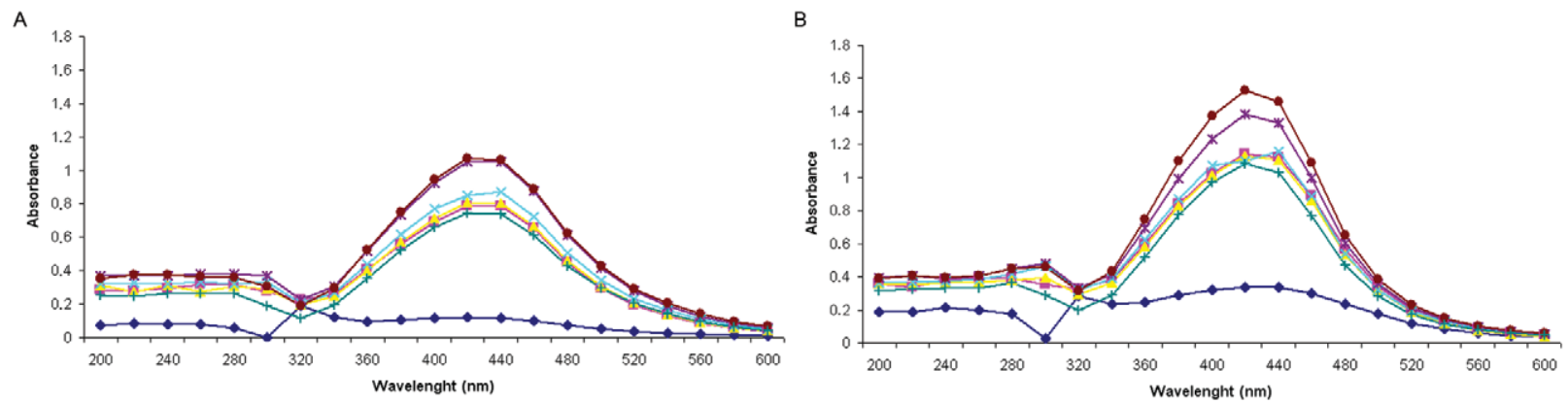

C

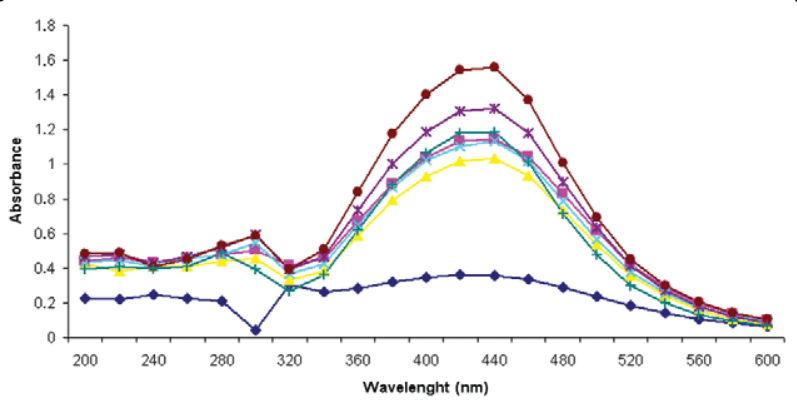

D

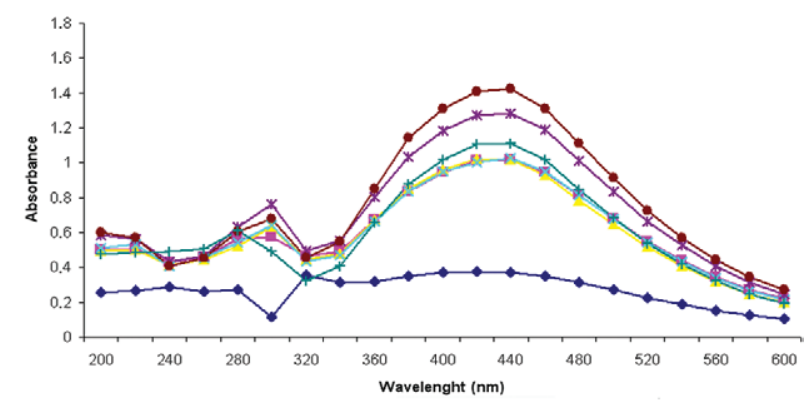

$$
\rightarrow-0 \rightarrow-3 \rightarrow-5 \rightarrow-10 \rightarrow-15 \rightarrow-30+60
$$

Days

Fig. 1: absorption spectrum from 20-600 nm of silver nanoparticles produced by aqueous extracts of Fusarium oxysporum and several concentrations of silver nitrate $\left(\mathrm{AgNO}_{3}\right)$ solutions at different times (0-60 days) after initiation of the reaction. $\mathrm{A}: 0.5 \mathrm{mM} \mathrm{AgNO} \mathrm{m}_{3} ; \mathrm{B}: 1.0 \mathrm{mM} \mathrm{AgNO}$; C: $1.5 \mathrm{mM} \mathrm{AgNO}_{3}$; D: $2.0 \mathrm{mM} \mathrm{AgNO}_{3}$. 
(Qian et al. 2013) are most efficient at producing SNPs. However, the majority of these studies used only a single concentration of $\mathrm{AgNO}_{3}$ solution $(1.0 \mathrm{mM})$ to produce SNPs. Our work further characterises the production of SNPs by a green synthesis method using aqueous extracts of $F$. oxysporum and different silver concentrations for up to 60 days. Spectrophotometric analyses revealed that concentrations below $1 \mathrm{mM} \mathrm{AgNO}_{3}$ produced fewer SNPs than concentrations $\geq 1 \mathrm{mM} \mathrm{AgNO}_{3}$. Interestingly, the use of concentrations $>1.0 \mathrm{mM}$ did not result in increased SNP production. With respect to the kinetics of SNP production, we found that increasing amounts of SNPs were produced for the first 30 days, followed by a decrease in production after 60 days. This finding indicates that the SNPs likely began to degrade after a prolonged incubation time. These changes in SNP production rates correlated with the appearance of needle-shaped SNPs, in addition to the previously observed spherical forms, on day 60 of the reactions. SNPs sizes could range from 1-100 $\mathrm{nm}$ and are characterised by a large surface area to volume ratio (Rai et al. 2009). The SNPs produced in this work were predominantly monodispersed and spherical, with diameters ranging from 1.9-64.9 $\mathrm{nm}$. Our data revealed that the optimal SNP production occurred when a $1.0 \mathrm{mM} \mathrm{AgNO}_{3}$ solution was used during three-five days into the reaction. In this case homogenous SNPs with approximate diameters of $10 \mathrm{~nm}$ were detected.

The reducing agent, reaction medium and SNP stabilisation are three key factors in the synthesis of metallic nanoparticles (Liu et al. 2009). According to Durán et al. (2005), reductases in the aqueous extracts of $F$. oxysporum are responsible for the reduction of $\mathrm{Ag}$ cations and subsequent SNP production. In addition, the SNP size, spherical form stability and dispersion may be mediated by an interaction between the SNPs and proteins present in the fungal extract (Durán et al. 2005). Furthermore, SNPs formed in solutions of complex organic molecules seem to be stabilised by a protective coating. Akaighe et al. (2011) reported that humic acid facilitate the generation of SNPs by reducing silver ions, which suggests that nanoparticles can exist in a natural environment. Humic acid appears to stabilise the nanoparticles by coating them and preventing their aggregation into a larger mass of silver (Akaighe et al. 2011). The fungal extract used in this work could also be considered a natural environment. This could explain the difference in the diameters of the agglomerates observed by AFM and TEM. TEM cannot resolve the large agglomerates of SNPs as well as AFM $(300 \pm 57 \mathrm{~nm})$. Furthermore, the TEM and SEM backscattered images only reveal the silver atomic nuclei.

TABLE I

Diameters of silver nanoparticles (SNPs)

produced from $1.0 \mathrm{mM}$ silver nitrate in aqueous extracts of Fusarium oxysporum at different reaction times

\begin{tabular}{lcc}
\hline & \multicolumn{2}{c}{$\begin{array}{c}\text { SNP diameter } \\
(\mathrm{nm})\end{array}$} \\
\cline { 2 - 3 } $\begin{array}{l}\text { Reaction time } \\
\text { (days) }\end{array}$ & Range & $\mathrm{X} \pm \mathrm{SD}$ \\
\hline 5 & $3.4-26.8$ & $13.2 \pm 4.2$ \\
10 & $4.8-56.6$ & $21.5 \pm 9.1$ \\
15 & $3.2-45.5$ & $13.5 \pm 7.6$ \\
30 & $4.8-64.9$ & $27.3 \pm 12.4$ \\
60 & $1.9-53.6$ & $10.4 \pm 10.1$ \\
\hline
\end{tabular}

SD: standard deviation; $\mathrm{X}$ : average.
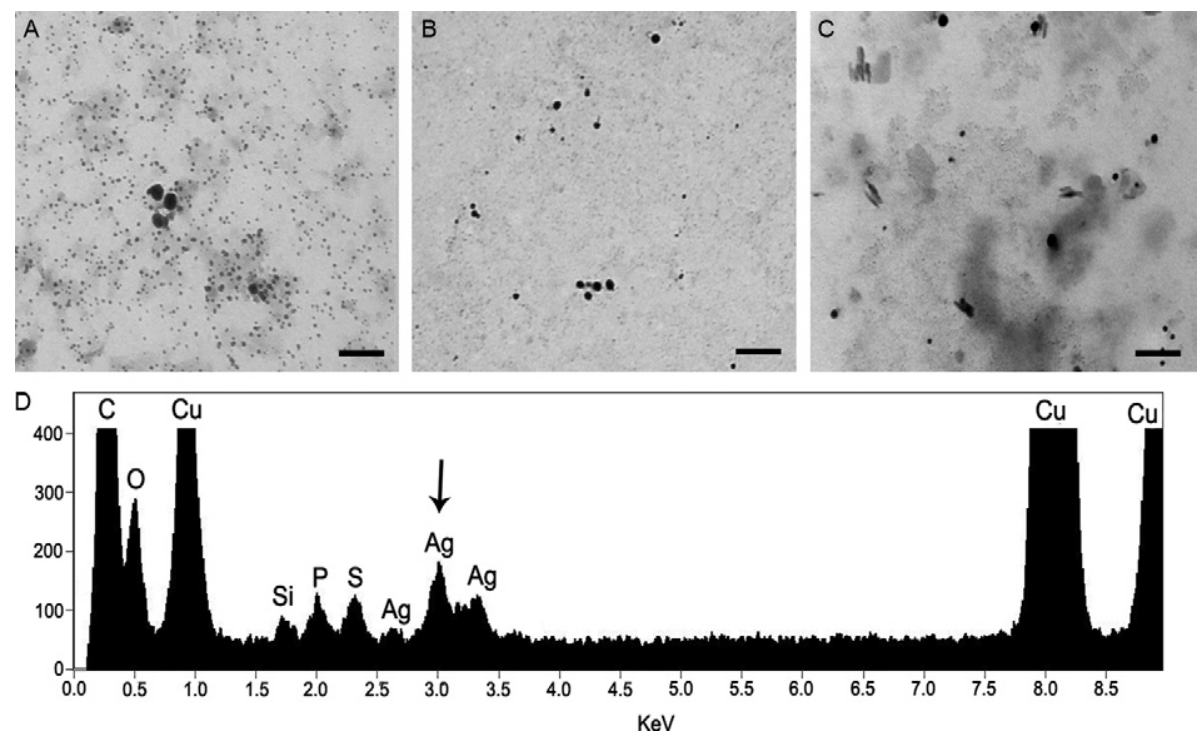

Fig. 3: transmission electron microscopy characterisation of silver nanoparticles produced during different reaction periods. A: five days; B: 30 days; C: 60 days. Bars $=200 \mathrm{~nm}$; D: the presence of silver atoms (black arrow) was confirmed by energy-dispersive X-ray spectroscopy; Ag: silver; C: carbon; Cu: copper; O: oxygen; P: phosphorus; S: sulphur; Si: silicon. 
Previous studies have shown that SNPs exhibit antimicrobial activity against different bacterial species, such as Shigella dysenteriae type I, Staphylococcus aureus, Citrobacter sp., Escherichia coli, Pseudomonas aeruginosa and Bacillus subtilis (Musarrat et al. 2010) and some fungal species, such as Trichophyton mentagrophytes and Candida spp (Keuk-Jun et al. 2008, Musarrat et al. 2010). In our work, we employed two different methods to determine the susceptibility of Candida spp and Cryptococcus spp. The disc diffusion method resulted in the formation of zones of growth inhibition ranging from $8-15 \mathrm{~mm}$ and with the broth microdilution assay we observed MICs of 0.42-1.68 $\mu \mathrm{g} / \mathrm{mL}$.

Similar results were previously reported by Musarrat et al. (2010) and Kaviya et al. (2011), who tested the effect of SNPs on bacteria and fungi using the same methods. Keuk-Jun et al. (2008) observed that the MICs of SNPs against $C$. albicans were lower $(2-4 \mu \mathrm{g} / \mathrm{mL})$ than against Candida non-albicans $(1-25 \mu \mathrm{g} / \mathrm{mL})$. Recently, Qian et
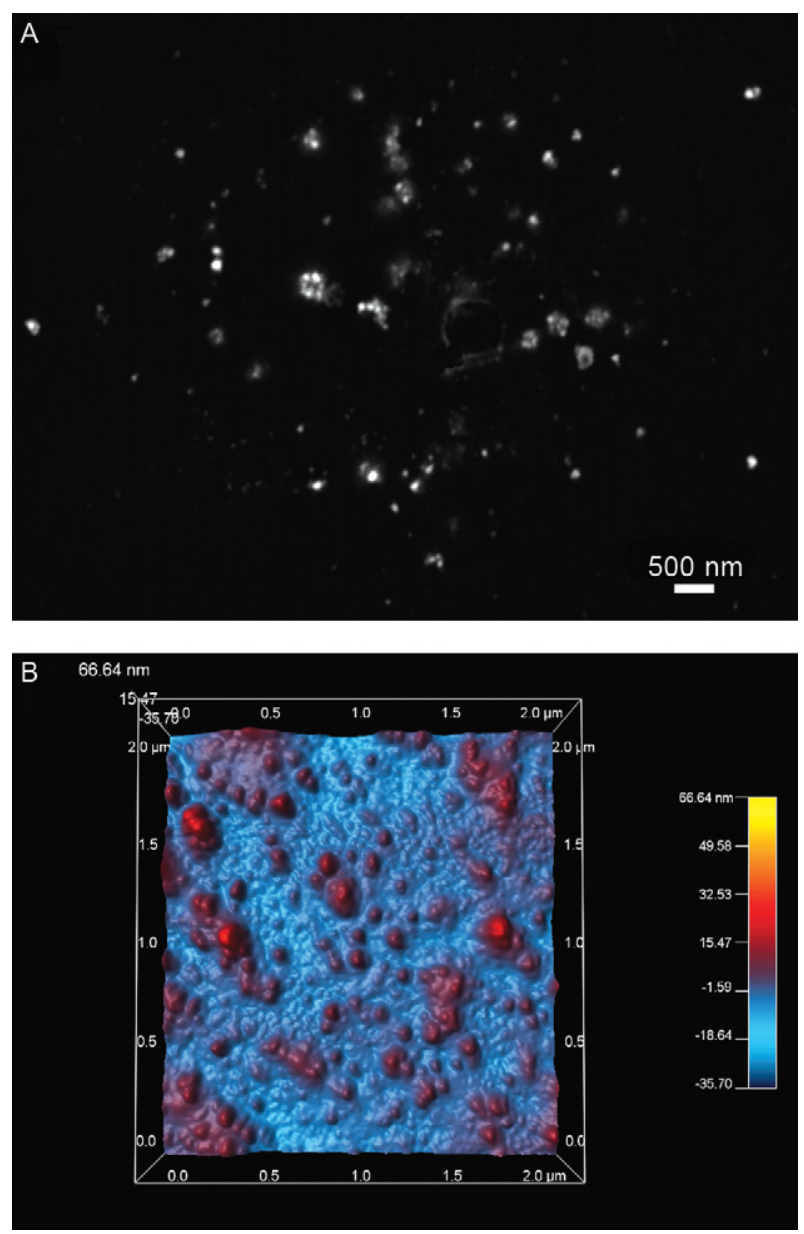

Fig. 4: microscopic characterisation of silver nanoparticles (SNPs) produced by aqueous extracts of Fusarium oxysporum and $1.0 \mathrm{mM}$ silver nitrate over five days. A: the presence of metallic particles was detected by scanning electron microscopy; B: the SNPS size and depth are indicated by the colour scale at the side of the atomic force microscopy micrograph. al. (2013) and Xu et al. (2013) observed SNPs antifungal activity against several fungi as Candida spp, Aspergillus spp, Fusarium spp, C. neoformans and Sporothrix schenckii presenting MIC values of $0.12-1 \mu \mathrm{g} / \mathrm{mL}$.

In our work, we observed similar MIC values for all Candida spp and Cryptococcus spp tested. However, the MFC values suggest that SNPs are fungistatic to C. parapsilosis and C. krusei and fungicidal to C. albicans, C. tropicalis, C. glabrata and Cryptococcus spp. Recently, Monteiro et al. (2011) reported an inhibitory effect of SNPs on C. albicans and C. glabrata biofilm formation due to reduced cell viability.

Our ultrastructural studies revealed that the yeast cell alterations induced by SNPs treatment largely occur in the cytoplasmic membrane and fungal CW. $C$. neoformans exposed to SNPs exhibit cytoplasmic leakage and apparent SNP retention in the polysaccharide capsule. To our knowledge, this is the first report that demonstrates the action of SNPs on C. neoformans cell envelope. Previous work has already demonstrated the ultrastructural effect on C. albicans yeast treated with SNPs (Kim et al. 2009). SNPs also presented inhibitory activity against $C$. albicans ( $\mathrm{MIC}=2 \mu \mathrm{g} / \mathrm{mL}$ ) and the action may exert the disrupting of the structure of the C. albicans $\mathrm{CW}$ and cytoplasm membrane and inhibit the normal budding process due to the destruction of the membrane integrity when yeasts were treated with SNPs concentration above MIC value $(170 \mu \mathrm{g} / \mathrm{mL}$ ) (Kim et al. 2009). Our work treated C. albicans yeasts with $\mathrm{MIC} / 2$ concentration of SNPs, however none ultrastructural alterations was observed by TEM technique. Interestingly, the MIC/2 value of SNPs was able to lead a change of cell envelop ultrastructure of $C$. neoformans.

Several other studies have demonstrated that the cell envelope constitutes the major target of SNP antimicrobial activity. Chwalibog et al. (2010) also demonstrated that the SNPs can self-assemble and interact with $C$. albicans and $S$. aureus cells, leading to the disintegration of CWs and cytoplasmic membranes and cytoplasm leakage. The effect of SNPs on S. aureus growth, morphology and CW integrity was investigated (Mirzajani et al. 2011), revealing bacterial growth inhibition, CW damage with peptidoglycan variations and release of muramic acid and accumulation of SNPs in the bacterial membrane at SNP concentrations greater than $8 \mu \mathrm{g} /$ $\mathrm{mL}$. Membrane disruption by SNPs is likely due to the production of reactive oxygen species (ROS), including free radicals that cause membrane lipid peroxidation. This membrane disruption allows the passage of SNPs into the cytoplasm, which causes subsequent damage of DNA and other phosphorus-containing compounds and impairs the respiratory chain and cell division (Rai et al. 2009). Recently, Hwang et al. (2012) showed SNPs possess antifungal effects through apoptosis. The treatment induced in C. albicans yeasts an accumulation of ROS, reduction in the mitochondrial membrane potential, phosphatidylserine externalisation, DNA and nuclear fragmentation and the activation of metacaspases.

In mammalian cells, cytotoxicity is related to a loss of cell viability, DNA fragmentation and subsequent 


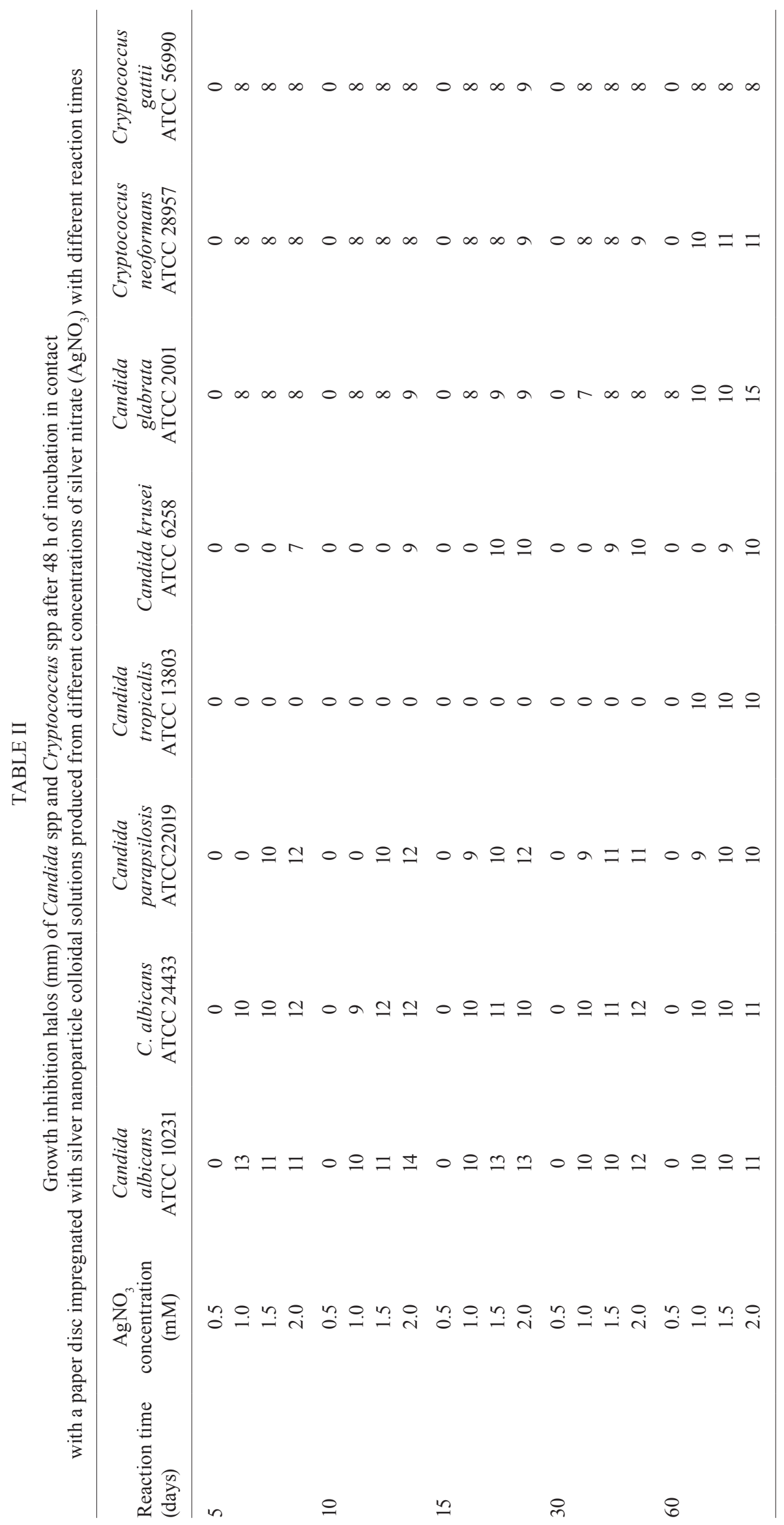


TABLE III

Minimum inhibitory concentration (MIC) and minimum fungicidal concentration (MFC) of silver nanoparticles (SNPs) for Candida spp and Cryptococcus spp concentrations

\begin{tabular}{lccc}
\hline Strains & $\begin{array}{c}\mathrm{MIC} \\
(\mu \mathrm{g} / \mathrm{mL})\end{array}$ & $\begin{array}{c}\mathrm{MFC} \\
(\mu \mathrm{g} / \mathrm{mL})\end{array}$ & MFC/MIC \\
\hline Cryptococcus neoformans ATCC 28957 & 0.42 & 0.42 & 1 \\
Cryptococcus gattii ATCC 56990 & 0.84 & 0.84 & 1 \\
Candida glabrata ATCC 2001 & 1.68 & 1.68 & 1 \\
Candida albicans ATCC 10231 & 1.68 & 3.4 & 2 \\
C. albicans ATCC 24433 & 1.68 & 3.4 & 2 \\
Candida tropicalis ATCC 13803 & 1.68 & 3.4 & 15 \\
Candida krusei ATCC 6258 & 0.84 & 13 & 15 \\
Candida parapsilosis ATCC 22019 & 0.84 & 13 & \\
\hline
\end{tabular}

apoptosis. Furthermore, the endoplasmic reticulum may play an important role in the response to oxidative stress-induced damage and is quite sensitive to oxidative damage (Zhang et al. 2012). Interestingly, biogenic SNPs seems to be generally less cyto/genotoxic in vivo compared with chemically synthesised nanoparticles. Furthermore, human cells were found to have a greater resistance to the toxic effects of SNPs in comparison with other organisms (Lima et al. 2012).

The antimicrobial effects of SNPs depend on their size and the rate of silver ion release. Smaller-sized particles exhibit greater activity due to their higher surface area to volume ratio (Morones et al. 2005). The ionic form of silver has been known for centuries to cure infectious diseases caused by various bacterial species, e.g., E. coli, S. aureus, Klebsiella sp. and Pseudomonas sp. (Chopra 2007, Rai et al. 2009). SNPs have an advantage over ionic silver because they exhibit reduced toxicity and greater antimicrobial potential (Ingle et al. 2008). The superior antimicrobial properties of SNPs compared to silver salts are due to the large surface area of the particles, which provides better contact with the microorganisms. In addition, the SNPs release silver ions directly into the bacteria, which enhance bactericidal activity (Rai et al. 2009).

Although in vitro and in vivo studies have indicated that SNPs are toxic to mammalian cells and that increased exposure of humans to SNPs entails potential risk, some studies have described broad applications of SNPs in the technological and medical fields (Ahmad et al. 2003, Chaloupka et al. 2010, Seil \& Webster 2012). However these applications remain largely unexplored. The production of SNPs using aqueous extracts of the fungus $F$. oxysporum is a potential candidate for lowcost and environmentally friendly production of stable and uniformly sized SNPs with anticandidal and anticryptococcal activities. In this regard, the results obtained in this work open several avenues of further study, such as purification and biochemical characterisation of reductases produced by $F$. oxysporum or development of an alternative SNP formulation that reduces the toxic-
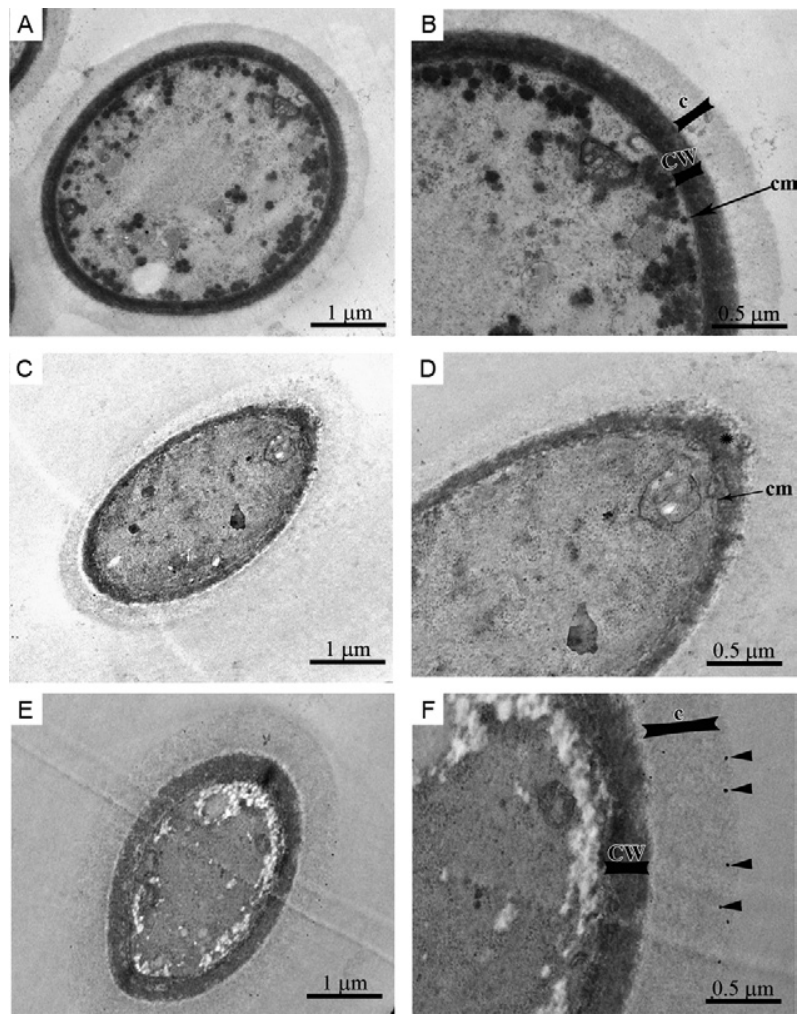

Fig. 5: morphological alterations of Cryptococcus neoformans treated with sub-inhibitory concentrations of silver nanoparticles (SNPs) $(0.21 \mu \mathrm{g} / \mathrm{mL})$ for $72 \mathrm{~h}$ at $35^{\circ} \mathrm{C}$. The untreated yeast exhibit a compact cell wall $(\mathrm{CW})$, continuous cytoplasmic membrane $(\mathrm{cm})$, homogeneous and electron-dense cytoplasm and a polysaccharide capsule (c) surrounding the cell (A, B). By contrast, yeasts treated with SNPs had a disrupted cytoplasmic membrane and $\mathrm{CW}$ (asterisk) and increased cell wall thickness $(\mathrm{CW})(\mathrm{C}, \mathrm{D})$. The SNPs appear to be retained in the polysaccharide capsule (F, black arrowhead).

ity of silver. In addition, the utilisation of silver metal in nanoparticulate form may be a new strategy for the treatment of fungal infection. 


\section{REFERENCES}

Ahamed M, AlSalhi MS, Siddiqui MKJ 2010. Silver nanoparticle applications and human health. Clin Chim Acta 411: 1841-1848.

Ahmad A, Mukherjee P, Senapati S, Mandal D, Khan MI, Kumar R, Sastry M 2003. Extracellular biosynthesis of silver nanoparticles using the fungus Fusarium oxysporum. Colloids Surf, B 28: $313-318$

Akaighe N, MacCuspie RI, Navarro DA, Aga DS, Banerjee S, Sohn M, Sharma VK 2011. Humic acid-induced silver nanoparticle formation under environmentally relevant conditions. Environ Sci Technol 45: 3895-3901.

Burgess LW 1981. General ecology of the fusaria. In PE Nelson, TA Toussoun, RJ Cook (eds.), Fusarium: diseases, biology and taxonomy, Pennsylvania State University Press, Philadelphia, p. $225-235$

Chaloupka K, Malam Y, Seifalian AM 2010. Nanosilver as a new generation of nanoproduct in biomedical applications. Trends Biotechnol 28: 580-588.

Chopra I 2007. The increasing use of silver-based products as antimicrobial agents: a useful development or a cause for concern? J Antimicrob Chemother 59: 587-590.

Chwalibog A, Sawosz E, Hotowy A, Szeliga J, Mitura S, Mitura K, Grodzik M, Orlowski P, Sokolowska A 2010. Visualization of interaction between inorganic nanoparticles and bacteria or fungi. Int J Nanomedicine 5: 1085-1094.

CLSI - Clinical and Laboratory Standards Institute 2004. Method for antifungal disk diffusion susceptibility testing of yeasts. Approved guideline M44-A, CLSI, Wayne, $23 \mathrm{pp}$.

CLSI - Clinical and Laboratory Standards Institute 2008. Reference method for broth dilution antifungal susceptibility testing of yeasts. Aproved standard, 3rd ed., M27-A3, CLSI, Wayne, 25 pp.

Durán N, Marcato PD, Alves OL, de Souza GIH, Esposito E 2005. Mechanistic aspects of biosynthesis of silver nanoparticles by several Fusarium oxysporum strains. J Nanobiotechnology 3: 1-8.

Fortin D, Beveridge TJ 2000. Mechanistic routes towards biomineral surface development. In E Baeuerlein (ed.), Biomineralization: from biology to biotechnology and medical application, WileyVCH Verlag, Germany, 294 pp.

Gade AK, Bonde P, Ingle AP, Marcato PD, Duran N, Rai MK 2008 Exploitation of Aspergillus niger for synthesis of silver nanoparticles. J Biobased Mater Bio 2: 243-247.

Hwang IS, Lee J, Hwang JH, Kim KJ, Lee DG 2012. Silver nanoparticles induce apoptotic cell death in Candida albicans through the increase of hydroxyl radicals. FEBS J 279: 1327-1338.

Ingle A, Gade A, Pierrat S, Sonnichsen C, Rai M 2008. Mycosynthesis of silver nanoparticles using the fungus Fusarium acuminatum and its activity against some human pathogenic bacteria. Curr Nanosci 4: 141-144.

Jillavenkatesa A, Dapkunas SJ, Lum LSH 2001. Particle size characterization, National Institute of Standards and Technology/Government Printing Office, Washington DC, 164 pp.
Kaviya S, Santhanalakshmi J, Viswanathan B, Muthumary J, Srinivasan K 2011. Biosynthesis of silver nanoparticles using Citrus sinensis peel extract and its antibacterial activity. Spectrochim Acta A Mol Biomol Spectrosc 79: 594-598

Keuk-Jun K, Sung WS, Moon SK, Choi JS, Kim JG, Lee DG 2008. Antifungal effect of silver nanoparticles on dermatophytes. J Microbiol Biotechnol 18: 1482-1484.

Kim KJ, Sung WS, Suh BK, Moon SK, Choi JS, Kim JG, Lee DG 2009. Antifungal activity and mode of action of silver nano-particles on Candida albicans. Biometals 22: 235-242.

Lima R, Seabra AB, Durán N 2012. Silver nanoparticles: a brief review of cytotoxicity and genotoxicity of chemically and biogenically synthesized nanoparticles. J Appl Toxicol 32: 867-879.

Liu J, He F, Gunn TM, Zhao D, Roberts CB 2009. Precise seed-mediated growth and size-controlled synthesis of palladium nanoparticles using a green chemistry approach. Langmuir 25: 7116-7128.

Mirzajani F, Ghassempour A, Aliahmadi A, Esmaeili MA 2011. Antibacterial effect of silver nanoparticles on Staphylococcus aureus. Res Microbiol 162: 542-549.

Monteiro DR, Gorup LF, Silva S, Negri M, Camargo ER, Oliveira R, Barbosa DB, Henriques M 2011. Silver colloidal nanoparticles: antifungal effect against adhered cells and biofilms of Candida albicans and Candida glabrata. Biofouling 27: 711-719.

Morones JR, Elechiguerra JL, Camacho A, Holt K, Kouri JB, Ramirez JT, Yacaman MJ 2005. The bactericidal effect of silver nanoparticles. Nanotechnology 16: 2346-2353.

Musarrat J, Dwivedi S, Singh BR, Al-Khedhairy AA, Naqvi AAA 2010. Production of antimicrobial silver nanoparticles in water extracts of the fungus Amylomyces rouxii strain KSU-09. Bioresour Technol 101: 8772-8776.

Narayanan KB, Sakthivel N 2010. Biological synthesis of metal nanoparticles by microbes. Adv Colloid Interface Sci 156: 1-13.

Nelson PE, Dignani MC, Anaissie EJ 1994. Taxonomy, biology and clinical aspects of Fusarium species. Clin Microbiol Rev 7: 479-504.

Qian Y, Yu H, He D, Yang H, Wang W, Wan X, Wang L 2013. Biosynthesis of silver nanoparticles by the endophytic fungus Epicoccum nigrum and their activity against pathogenic fungi. Bioprocess Biosyst Eng: doi 10.1007/s00449-013-0937-z.

Quester K, Avalos-Borja M, Castro-Longoria E 2013. Biosynthesis and microscopic study of metallic nanoparticles. Micron: doi: 10.1016/j.micron.2013.07.003

Rai M, Yadav A, Gade A 2009. Silver nanoparticles as a new generation of antimicrobials. Biotechnol Adv 27: 76-83.

Seil JT, Webster TJ 2012. Antimicrobial applications of nanotechnology: methods and literature. Int J Nanomedicine 7: 2767-2781.

Xu Y, Gao C, Li X, He Y, Zhou L, Pang G, Sun S 2013. In vitro antifungal activity of silver nanoparticles against ocular pathogenic filamentous fungi. J Ocul Pharmacol Ther 29: 270-274.

Zhang R, Piao MJ, Kim KC, Kim AD, Choi JY, Choi J, Hyun JW 2012. Endoplasmic reticulum stress signaling is involved in silver nanoparticles-induced apoptosis. Int J Biochem Cell Biol 44: 224-232. 